\title{
Comparative effectiveness of $\beta$-lactam versus vancomycin empiric therapy in patients with methicillin-susceptible Staphylococcus aureus (MSSA) bacteremia
}

\author{
Davie Wong ${ }^{1 *}$, Titus Wong ${ }^{2,3}$, Marc Romney ${ }^{2,4}$ and Victor Leung ${ }^{2,4}$
}

\begin{abstract}
Background: Vancomycin may be inferior to $\beta$-lactams for the empiric treatment of methicillin-susceptible Staphylococcus aureus (MSSA) bacteremia. We compared empiric $\beta$-lactams to vancomycin to assess clinical outcomes in patients with MSSA bacteremia.

Methods: We conducted a retrospective cohort study of adult inpatients with their first episode of MSSA bacteremia at two tertiary care hospitals in Vancouver, Canada, between 2007 and 2014. Exposure was either empiric $\beta$-lactam with or without vancomycin or vancomycin monotherapy. All patients received definitive treatment with cloxacillin or cefazolin. The primary outcome was 28 -day mortality. Secondary outcomes were 90-day mortality, duration of bacteremia, and hospital length-of-stay. Outcomes were adjusted using multivariable logistic regression.
\end{abstract}

Results: Of 669 patients identified, 255 met inclusion criteria $(\beta$-lactam $=131$, vancomycin $=124)$. Overall 28-day mortality was $7.06 \%(n=18)$. There were more cases of infective endocarditis in the $\beta$-lactam than in the vancomycin group [24 (18.3\%) vs 12 (9.7\%), $p=0.05]$. Adjusted mortality at 28 days was similar between the two groups (OR 0.85; $95 \% \mathrm{Cl} 0.27-2.67$ ). The duration of bacteremia was longer in the vancomycin group (97.1 vs $70.7 \mathrm{~h}, p=0.007$ ). Transition to cloxacillin or cefazolin occurred within a median of $68.3 \mathrm{~h}$ in the vancomycin group.

Conclusions: Empiric $\beta$-lactams was associated with earlier clearance of bacteremia by a median of 1 day compared to vancomycin. Future prospective studies are needed to confirm our findings.

Keywords: Staphylococcus aureus, Bacteremia, Methicillin-susceptible, Vancomycin, Beta-lactam, Empiric therapy

\section{Background}

Staphylococcus aureus is the leading cause of bacteremia and carries a mortality of $20-30 \%$ in the twentyfirst century $[1,2]$. Empiric vancomycin is commonly prescribed when $S$. aureus is isolated from a blood culture but antimicrobial susceptibilities are not yet known, because up to $50-60 \%$ of bloodstream isolates are methicillin-resistant $S$. aureus (MRSA) at some centres [3-8]. However, vancomycin is inferior to semi-synthetic

\footnotetext{
${ }^{*}$ Correspondence: davie1985@hotmail.com

1 PGY-V Infectious Diseases Residency Training Program, Vancouver General Hospital, University of British Columbia, D 452 Heather Pavilion, 2733 Heather Street, Vancouver, BC V5Z 1M9, Canada

Full list of author information is available at the end of the article
}

anti-Staphylococcal penicillins (e.g., cloxacillin) and first generation cephalosporins (e.g., cefazolin) for the definitive treatment of methicillin-susceptible $S$. aureus (MSSA) bacteremia [9-11]. Cloxacillin and cefazolin are equally efficacious in treating MSSA bacteremia and are the optimal agents against MSSA [10]. Vancomycin is associated with higher rates of infection-related mortality, re-infection and bacteriologic failure compared to cloxacillin or cefazolin in the definitive treatment of MSSA bacteremia [9, 12-15]. Whether vancomycin is inferior to $\beta$-lactams for empiric therapy remains to be fully elucidated. Early studies suggested that empiric vancomycin was associated with worse outcomes compared to empiric $\beta$-lactam therapy $[3,16,17]$, but more recent 
data did not demonstrate any differences [15]. Although controversial, some experts recommend the addition of a $\beta$-lactam agent to empiric therapy to provide optimal coverage for MSSA in patients at the highest risk of morbidity and mortality from $S$. aureus bacteremia (SAB) [18]. Currently, no studies have compared empiric $\beta$-lactam to vancomycin in patients with MSSA bacteremia who are transitioned to cloxacillin or cefazolin for definitive therapy. We assessed if empiric $\beta$-lactam with or without vancomycin compared to vancomycin alone was associated with differences in clinical outcomes in patients with MSSA bacteremia who received definitive therapy with cloxacillin or cefazolin.

\section{Methods}

\section{Patients}

We performed a retrospective cohort study of adult inpatients aged 18 and older diagnosed with their first episode of MSSA bacteremia at two tertiary care hospitals in Vancouver, Canada, between January 2007 and December 2014, inclusive. Both hospitals are large academic institutions (955 and 435 beds) affiliated with the University of British Columbia that are served by infectious diseases physicians who share similar treatment strategies for SAB. Consecutive patients were included if they received definitive therapy with either cloxacillin or cefazolin. Patients were excluded if there was missing data for 28-day mortality, no empiric therapy was administered, death occurred within $24 \mathrm{~h}$ following diagnosis of bacteremia, or polymicrobial bacteremia. Patients were stratified based on empiric treatment with $\beta$-lactams or vancomycin. The $\beta$-lactam group received one or more of cloxacillin, cefazolin, $\beta$-lactam/ $\beta$-lactamase inhibitor combination, a third generation cephalosporin or a carbapenem, with or without vancomycin. The vancomycin group was not exposed to any $\beta$-lactams until the start of definitive therapy. In both groups, other antimicrobials may have been prescribed during empiric and definitive therapy.

\section{Definitions}

Bacteremia was defined as the isolation of MSSA from one or more blood culture bottles. Bacteremia identified within $48 \mathrm{~h}$ of hospital admission was considered community-onset, while bacteremia diagnosed after more than $48 \mathrm{~h}$ of hospital admission was deemed hospital-onset. Immunocompromised state was present if any of the following were described: neutropenia $\left(\leq 1.5 \times 10^{9} / \mathrm{L}\right)$, congenital immune deficiencies, or use of immunosuppressants (TNF- $\alpha$ inhibitors, prednisone $\geq 10 \mathrm{mg} /$ day or its equivalent, cancer chemotherapy, methotrexate, cyclophosphamide, mycophenolate mofetil, calcineurin inhibitors, mTOR inhibitors, azathioprine). Definite infective endocarditis was diagnosed using the modified Duke criteria [19]. The source of bacteremia was either stated explicitly or inferred as the most likely source based on available clinical data and microbiological results. Metastatic complications included infections that occurred distant from the presumed primary source such as septic emboli, mycotic aneurysms, osteoarticular infections, and distant abscesses. Surgical source control included only procedures performed in the operating theatre. Empiric therapy began with the first dose of empiric antibiotics and ended with the start of definitive therapy. Definitive therapy began when antimicrobial susceptibilities were released and one of the following treatments was prescribed: (1) cloxacillin or cefazolin (2) discontinuation of other empiric antibiotics for patients already on cloxacillin or cefazolin empirically, or (3) continuation of empiric cloxacillin or cefazolin. Definitive therapy ended when cloxacillin or cefazolin was stopped. Time to receipt of antibiotics was measured from the time of obtaining the first positive blood culture to the time of the first dose of antibiotic. If a patient was already on antibiotics at the time of the first positive blood culture, the time to receipt of antibiotics was zero.

\section{Outcomes}

Our primary outcome was 28-day all-cause mortality. Secondary outcomes were 90-day all-cause mortality, duration of bacteremia, and hospital length-of-stay (LOS). Time to mortality was measured from the date of the first positive blood culture to the date of death. Duration of bacteremia was the time difference between the first positive blood culture and the first negative blood culture. Patients without follow-up blood cultures were excluded from the analysis for duration of bacteremia. Hospital LOS was measured from the date of the first positive blood culture for MSSA to the date of discharge. Patients who did not survive to hospital discharge were excluded from the hospital LOS analysis.

\section{Data extraction}

Patients with MSSA bacteremia were extracted from the medical microbiology laboratory information systems and medical records were reviewed. A single reviewer collected data on patient demographics and comorbidities, blood culture results and antimicrobial therapy.

\section{Statistical analysis}

Our predicted mortality difference between the $\beta$-lactam and vancomycin group was $15 \%$ based on a previous study [17]. We estimated a sample size of 100 for each group to capture a $15 \%$ difference in mortality, assuming a mortality rate of 25 and $10 \%$ in the vancomycin and $\beta$-lactam group respectively, with $80 \%$ power at a 
two-tailed alpha level of 0.05 . Baseline categorical variables were described as counts and percentages, and differences between groups were assessed with Chi square or Fisher's exact tests. Continuous variables were presented as means and standard deviations, or medians and interquartile range. Differences between groups were assessed using parametric t-tests or non-parametric Mann-Whitney U tests, as appropriate. Logistic regression methods were used to model the odds ratio of death in the $\beta$-lactam compared to vancomycin group. In order to reduce the small sample size bias, Firth correction method was applied to 28 - and 90-day mortality [20]. Linear regression model was conducted for hospital LOS and duration of bacteremia. The two outcomes were $\log$-transformed in the analysis to improve normality of the distribution of residuals. All models were adjusted for age, age-adjusted Charlson-comorbidity index (CCI) [21], infectious diseases consultation, infective endocarditis and time to receipt of empiric antibiotics. The duration of bacteremia was further adjusted for surgical source control. All analyses were performed using the SAS 9.4 software.

\section{Ethics}

The study was approved by the research ethics board at the University of British Columbia, and received institutional approval from Vancouver Coastal Health and Providence Healthcare.

\section{Results}

We identified 669 patients with MSSA bacteremia between January 2007 and December 2014, inclusive (Fig. 1). We excluded 414 patients primarily because $60.4 \%$ did not receive cloxacillin or cefazolin for definitive therapy. These patients either remained on broadspectrum antimicrobials or received vancomycin for

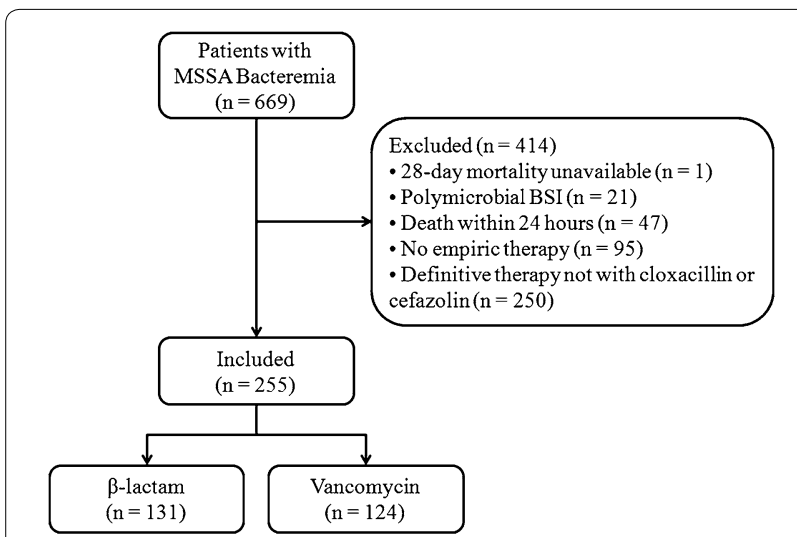

Fig. 1 Patient enrollment process. MSSA methicillin-susceptible $S$. aureus, $B S /$ bloodstream infection definitive therapy due to suspected or confirmed penicillin allergy. Another $22.9 \%$ were not started on empiric therapy. Our cohort consisted of $66.3 \%$ males and $74.1 \%$ of patients had community-onset bacteremia (Table 1). Compared to the $\beta$-lactam group, patients in the vancomycin group were older (mean age 59.4 vs 53.2 years, $p=0.005$ ), had more medical comorbidities (median CCI 4 vs $3, p=0.001$ ), and were diagnosed with a greater proportion of hospital-onset bacteremia (32.3 vs $19.8 \%$, $p=0.03)$. Infectious diseases consultation was obtained in most cases (69.8\%), but tended to be higher in the $\beta$-lactam $(74.8 \%)$ than in the vancomycin group $(64.5 \%)$. The most common sources of bacteremia in our cohort were unknown $(27.1 \%)$, skin and soft tissue infections (18.4\%), peripheral or central venous catheters (16.5\%) and injection drug use (15.7\%). Infective endocarditis was diagnosed more frequently and surgical source control was achieved more often in the $\beta$-lactam (18.3 and $25.2 \%$ respectively) compared to the vancomycin group (9.7 and $15.3 \%$ respectively). The prevalence of infective endocarditis was $14.1 \%$.

In the $\beta$-lactam group, the use of multiple $\beta$-lactam antibiotics reflects changes made during empiric therapy, but cloxacillin or cefazolin was continued until the start of definitive therapy. One patient in the vancomycin group received only a partial dose of vancomycin and was counted as not having received it. There was a greater delay in receipt of empiric antimicrobials in the vancomycin group (median 20.9 vs $2 \mathrm{~h}, p<0.001$ ).Transition to cloxacillin or cefazolin occurred within a median of $68.3 \mathrm{~h}$ in the vancomycin group. Among the subgroup of patients who received combination therapy with $\beta$-lactam plus vancomycin (88/131), 3rd generation cephalosporins $(39.8 \%)$ and piperacillin-tazobactam $(34.1 \%)$ were the most common empiric $\beta$-lactams prescribed initially (Table 2). Initiation of cloxacillin or cefazolin during the empiric period was delayed in the combination subgroup compared to the subgroup that received $\beta$-lactam monotherapy (median 23.1 vs $6.5 \mathrm{~h}, p=0.001$ ). The combination subgroup had a higher Pitt bacteremia score (median 1 vs $0, p=0.01$ ), received more infectious diseases consultations (80.7 vs $62.8 \%, p=0.03$ ), and experienced more metastatic complications ( 37.5 vs $16.3 \%, p=0.02$ ) than the $\beta$-lactam monotherapy subgroup. Hospital LOS was shorter in patients who received $\beta$-lactam monotherapy (median 14 vs 22 days, $p=0.02$ ).

The adjusted odds ratio of death at 28 and 90 days between the $\beta$-lactam and vancomycin group was 0.85 (95\% CI $0.27-2.67)$ and $0.88(0.36-2.17)$ respectively (Table 3). The overall 28- and 90-day mortality was 18 (7.06 \%) and $36(14.1 \%)$ respectively. Among patients with infective endocarditis, 28- and 90-day mortality was $2(8.33 \%)$ and $3(12.5 \%)$ in the $\beta$-lactam group and 
0 and $1(8.33 \%)$ in the vancomycin group. No mortality differences were observed between the two hospitals. The duration of bacteremia was shorter in the $\beta$-lactam than in the vancomycin group (median 70.7 vs $97.1 \mathrm{~h}$, $p=0.007)$ with an adjusted ratio of mean of 0.77 (95\% CI 0.62-0.95). Hospital LOS was similar between the two groups with an adjusted ratio of mean of $0.86(95 \% \mathrm{CI}$ $0.66-1.10)$.

Table 1 Comparison of baseline characteristics and clinical outcomes of patients with methicillin-susceptible S. aureus bacteremia who received empiric antimicrobial therapy with $\beta$-lactams or vancomycin

\begin{tabular}{|c|c|c|c|}
\hline Patient characteristics & $\beta$-lactam $^{a}(n=131)$ & Vancomycin $^{a}(n=124)$ & $p$ value \\
\hline Age (mean \pm standard deviation in years) & $53.2 \pm 16.5$ & $59.4 \pm 18.3$ & 0.005 \\
\hline Males & $83(63.4)$ & $86(69.4)$ & 0.31 \\
\hline Community-onset & $105(80.2)$ & $84(67.8)$ & 0.03 \\
\hline Hospital-onset & $26(19.8)$ & $40(32.3)$ & 0.03 \\
\hline HIV infection & $11(8.4)$ & $8(6.5)$ & 0.55 \\
\hline Hepatitis C infection & $29(22.1)$ & $28(22.6)$ & 0.93 \\
\hline Immunocompromised & $14(10.7)$ & $10(8.1)$ & 0.47 \\
\hline Alcohol or illicit drug abuse & $41(31.3)$ & $38(30.6)$ & 0.91 \\
\hline Intravenous drug use & $29(22.1)$ & $29(23.4)$ & 0.81 \\
\hline Charlson comorbidity index (median with IQR) & $3(1.0-6.0)$ & $4(1.0-7.0)$ & 0.001 \\
\hline Pitt bacteremia score (median with IQR) & $1(0-2)$ & $1(0-2)$ & 0.17 \\
\hline Infectious diseases consultation & $98(74.8)$ & $80(64.5)$ & 0.07 \\
\hline \multicolumn{4}{|l|}{ Source of bacteremia } \\
\hline Central or peripheral line & $20(15.3)$ & $22(17.7)$ & 0.62 \\
\hline Skin and soft tissue & $31(23.7)$ & $16(12.9)$ & 0.04 \\
\hline Intravenous drug use & $21(16.0)$ & $19(15.3)$ & 1.00 \\
\hline Bone or joint infection & $18(13.7)$ & $10(8.1)$ & 0.16 \\
\hline Lung & $4(3.1)$ & $3(2.4)$ & 1.00 \\
\hline Other & $12(9.2)$ & $10(8.1)$ & 0.83 \\
\hline Unknown & $25(19.1)$ & $44(35.5)$ & 0.005 \\
\hline Infective endocarditis & $24(18.3)$ & $12(9.7)$ & 0.05 \\
\hline Metastatic complications & $40(30.5)$ & $27(21.8)$ & 0.11 \\
\hline Surgical source control & $33(25.2)$ & $19(15.3)$ & 0.05 \\
\hline Recurrent infection at 6 months & $4(3.1)$ & $4(3.2)$ & 1.00 \\
\hline \multicolumn{4}{|l|}{ Empiric antimicrobials } \\
\hline Vancomycin & $88(67.2)$ & $123(99.2)$ & $<0.001$ \\
\hline Daptomycin & $2(1.5)$ & 0 & 0.50 \\
\hline Linezolid & $2(1.5)$ & 0 & 0.50 \\
\hline Cloxacillin or cefazolin & $131(100)$ & 0 & $<0.001$ \\
\hline 3rd generation cephalosporin & $40(30.5)$ & 0 & $<0.001$ \\
\hline Piperacillin-tazobactam & $33(25.2)$ & 0 & $<0.001$ \\
\hline Ticarcillin-clavulanic acid & $3(2.3)$ & 0 & 0.25 \\
\hline Carbapenem & $2(1.5)$ & 0 & 0.50 \\
\hline Other $^{b}$ & $47(35.9)$ & $66(53.2)$ & 0.01 \\
\hline Blood culture time to positivity (median hours with IQR) & $20(16.5-24.9)$ & $18.3(15.9-23.5)$ & 0.17 \\
\hline Duration of empiric therapy (median hours with IQR) & $54(42.0-69.0)$ & $48(29.6-75.8)$ & 0.28 \\
\hline Duration of definitive therapy (median days with IQR) & $31.5(13.0-42.0)$ & $28(10.0-42.0)$ & 0.17 \\
\hline Time to receipt of empiric therapy (median hours with IQR) & $2(0-7)$ & $20.9(4.2-28.3)$ & $<0.001$ \\
\hline Time to receipt of $\beta$-lactam (median hours with IQR) & $3(0.2-16.3)$ & $68.2(51.5-95.4)$ & $<0.001$ \\
\hline Time to receipt of cloxacillin or cefazolin (median hours with IQR) & $21.0(4.4-31.2)$ & $68.3(51.6-95.4)$ & $<0.001$ \\
\hline \multicolumn{4}{|l|}{ Primary outcome } \\
\hline 28-day mortality & $7(5.3)$ & $11(8.9)$ & 0.27 \\
\hline
\end{tabular}


Table 1 continued

\begin{tabular}{|c|c|c|c|}
\hline Patient characteristics & $\beta$-lactam $^{a}(n=131)$ & Vancomycin $^{a}(n=124)$ & $p$ value \\
\hline \multicolumn{4}{|l|}{ Secondary outcomes } \\
\hline 90-day mortality & $14(10.7)$ & $22(17.7)$ & 0.11 \\
\hline Duration of bacteremia (median hours with IQR) ${ }^{c}$ & $70.7(46.9-119)$ & $97.1(61.6-148)$ & 0.007 \\
\hline$\geq 3$ days & $58(50)$ & $72(63.2)$ & 0.047 \\
\hline Hospital length of stay (median days with IQR) ${ }^{d}$ & $17(11-36)$ & $17(12-36.5)$ & 0.84 \\
\hline \multicolumn{4}{|l|}{$I Q R$ interquartile range } \\
\hline \multicolumn{4}{|c|}{ a Variables are displayed as counts and percentages in parentheses unless otherwise specified } \\
\hline
\end{tabular}

\section{Discussion}

The goal of our study was to assess if empiric $\beta$-lactams with or without vancomycin compared to vancomycin alone was associated with differences in outcomes in patients with MSSA bacteremia. We found no differences in all-cause mortality at 28 and 90 days, or hospital LOS between these two groups. Clearance of bacteremia was delayed by a median of 1 day in the vancomycin group. However, this outcome may have been confounded by the earlier receipt of empiric antibiotics in the $\beta$-lactam group (median $2 \mathrm{~h}$ ). When we analyzed a subset of patients from the vancomycin group $(n=43)$ whose median time to receipt of empiric antibiotics was $1.97 \mathrm{~h}$, the duration of bacteremia was still longer compared to the $\beta$-lactam group, but just shy of statistical significance ( 95.1 vs $70.7 \mathrm{~h}, p=0.06$ ), likely because of the reduced sample size. Therefore, it does not appear that time to receipt of empiric therapy had a major impact on time to clearance of bacteremia in our study. Despite the high prevalence of MRSA at both of our institutions (25 and $38 \%$ ), only $67.2 \%$ of patients in the $\beta$-lactam group received vancomycin empirically as well. Perhaps the awareness of MRSA was low among some treating clinicians or patients who did not receive empiric vancomycin were judged to be at low risk for MRSA infection.

Interestingly, the differential time delay in receipt of empiric antimicrobials was unexpected. Patients in the $\beta$-lactam group generally received antibiotics well before the blood culture became positive, while patients in the vancomycin group tended to receive antibiotics shortly after the blood culture turned positive. The reason for this observation is likely multifactorial. First, $\beta$-lactam patients generally had more identifiable sources of bacteremia (i.e. more skin and soft tissue infections). Second, the higher prevalence of community-onset bacteremia suggests these patients may have had their first medical contact with the emergency department where sepsis protocols facilitated timely administration of antibiotics.
In the $\beta$-lactam group, patients who received empiric $\beta$-lactam plus vancomycin had a higher Pitt bacteremia score, experienced more metastatic complications and stayed in hospital longer than those who received empiric $\beta$-lactam monotherapy. The greater severity of illness in this combination subgroup may explain the initial use of broad-spectrum $\beta$-lactams (ceftriaxone or piperacillin-tazobactam), with subsequent de-escalation to cloxacillin or cefazolin during the empiric period by the infectious diseases consultant when $S$. aureus was identified in the blood culture. De-escalation occurred within a median of $23.1 \mathrm{~h}$, which follows the time to positivity of the first blood culture (median $20 \mathrm{~h}$ ). Despite differences in baseline characteristics and antimicrobials prescribed, mortality rates and time to clearance of bacteremia were similar between these subgroups.

Our study outcomes were similar to those reported in the literature. The overall 28- and 90-day mortality in our study was low at $18(7.06 \%)$ and $36(14.1 \%)$ respectively, but is within the range of 3.6-51.7 \% described in a meta-analysis of patients with MSSA bacteremia from catheter-related infections and infective endocarditis by Cosgrove et al. [22]. Definite infective endocarditis was diagnosed in 36 (14.1\%) of our patients, which is similar to rates reported in previous studies [10, 14, 16, 23, 24].

The median duration of bacteremia was longer in the vancomycin compared to the $\beta$-lactam group (4 vs 3 days) in our study. In a similar study by Khatib et al. [3], clearance of bacteremia was delayed (duration $\geq 3$ days) more often in patients who received empiric vancomycin $(57.6 \%)$ compared to those who received empiric $\beta$-lactams $(37.5 \%)$. They reported no difference in allcause or attributable mortality between groups.

We did not find any differences in mortality between treatment groups in our study. In contrast, Lodise et al. [17] demonstrated that empiric $\beta$-lactam was associated with lower infection-related mortality than with empiric vancomycin monotherapy (11.4 vs $39.3 \%, p=0.005)$ 
Table 2 Comparison of baseline characteristics and clinical outcomes of patients with methicillin-susceptible S. aureus bacteremia who received empiric combination therapy with $\beta$-lactam plus vancomycin or empiric $\beta$-lactam monotherapy

\begin{tabular}{|c|c|c|c|}
\hline Patient characteristics & $\begin{array}{l}\beta \text {-lactam plus vancomycin }{ }^{a} \\
(n=88)\end{array}$ & $\begin{array}{l}\beta \text {-lactam monotherapy } \\
(\mathrm{n}=43)\end{array}$ & $p$ value \\
\hline Age (mean \pm standard deviation in years) & $50.5 \pm 16.4$ & $58.9 \pm 16.6$ & 0.006 \\
\hline Males & $53(60.2)$ & $30(69.8)$ & 0.34 \\
\hline Community-onset & $74(84.1)$ & $31(72.1)$ & 0.16 \\
\hline Hospital-onset & $14(15.9)$ & $12(27.9)$ & 0.16 \\
\hline HIV infection & $9(10.2)$ & $2(4.7)$ & 0.34 \\
\hline Hepatitis C infection & $19(21.6)$ & $10(23.3)$ & 0.83 \\
\hline Immunocompromised & $8(9.1)$ & $6(14.0)$ & 0.39 \\
\hline Alcohol or illicit drug abuse & $29(33.0)$ & $12(27.9)$ & 0.69 \\
\hline Intravenous drug use & $21(23.9)$ & $8(18.6)$ & 0.65 \\
\hline Charlson comorbidity index (median with IQR) & $2(1-4)$ & $3(1-5)$ & 0.07 \\
\hline Pitt bacteremia score (median with IQR) & $1(0-2)$ & $0(0-1)$ & 0.01 \\
\hline Infectious diseases consultation & $71(80.7)$ & $27(62.8)$ & 0.03 \\
\hline \multicolumn{4}{|l|}{ Source of bacteremia } \\
\hline Central or peripheral line & $12(13.6)$ & $8(18.6)$ & 0.45 \\
\hline Skin and soft tissue & $20(22.7)$ & $11(25.6)$ & 0.83 \\
\hline Intravenous drug use & $16(18.2)$ & $5(11.6)$ & 0.45 \\
\hline Bone or joint infection & $10(11.4)$ & $8(18.6)$ & 0.29 \\
\hline Lung & $2(2.3)$ & $2(4.7)$ & 0.60 \\
\hline Other & $9(10.2)$ & $3(7.0)$ & 0.75 \\
\hline Unknown & $19(21.6)$ & $6(14.0)$ & 0.35 \\
\hline Infective endocarditis & $19(21.6)$ & $5(11.6)$ & 0.23 \\
\hline Metastatic complications & $33(37.5)$ & $7(16.3)$ & 0.02 \\
\hline Surgical source control & $22(25)$ & $11(25.6)$ & 1.00 \\
\hline Recurrent infection at 6 months & $2(2.3)$ & $2(4.7)$ & 0.60 \\
\hline \multicolumn{4}{|l|}{ Empiric antimicrobials } \\
\hline Daptomycin & 0 & $2(4.7)$ & 0.11 \\
\hline Linezolid & $1(1.1)$ & $1(2.3)$ & 0.55 \\
\hline Cloxacillin or cefazolin & $88(100)$ & $43(100)$ & 1.00 \\
\hline 3rd generation cephalosporin & $35(39.8)$ & $5(11.6)$ & 0.001 \\
\hline Piperacillin-tazobactam & $30(34.1)$ & $3(7.0)$ & $<0.001$ \\
\hline Ticarcillin-clavulanic acid & $2(2.3)$ & $1(2.3)$ & 1.00 \\
\hline Carbapenem & $1(1.1)$ & $1(2.3)$ & 0.55 \\
\hline Other ${ }^{b}$ & $27(30.7)$ & $20(46.5)$ & 0.08 \\
\hline Blood culture time to positivity (median hours with IQR) & $20(16.0-24.0)$ & $20.7(18-27.3)$ & 0.07 \\
\hline Duration of empiric therapy (median hours with IQR) & $54.1(43.0-71.7)$ & $51.7(38-64.5)$ & 0.18 \\
\hline Duration of definitive therapy (median days with IQR) & $38(16-43)$ & $23(12-40)$ & 0.08 \\
\hline Time to receipt of empiric therapy (median hours with IQR) & $1.91(0-6.17)$ & $3.42(0.58-17.2)$ & 0.19 \\
\hline Time to receipt of $\beta$-lactam (median hours with IQR) & $2.88(0.21-15.1)$ & $3.42(0.58-17.2)$ & 0.87 \\
\hline $\begin{array}{l}\text { Time to receipt of cloxacillin or cefazolin (median hours with } \\
\text { IQR) }\end{array}$ & $23.1(13.0-31.7)$ & $6.5(1.5-22.1)$ & 0.001 \\
\hline \multicolumn{4}{|l|}{ Primary outcome } \\
\hline 28-day mortality & $5(5.7)$ & $2(4.7)$ & 1.00 \\
\hline
\end{tabular}


Table 2 continued

\begin{tabular}{|c|c|c|c|}
\hline Patient characteristics & $\begin{array}{l}\beta \text {-lactam plus vancomycin } \\
(n=88)\end{array}$ & $\begin{array}{l}\beta \text {-lactam monotherapy } \\
(n=43)\end{array}$ & $p$ value \\
\hline \multicolumn{4}{|l|}{ Secondary outcomes } \\
\hline 90-day mortality & $10(11.4)$ & $4(9.3)$ & 1.00 \\
\hline Duration of bacteremia (median hours with $\mathrm{IQR})^{\mathrm{C}}$ & $71.4(50.3-126.9)$ & $68.5(36.4-115.8)$ & 0.35 \\
\hline$\geq 3$ days & $41(46.6)$ & $17(39.5)$ & 0.46 \\
\hline Hospital length of stay (median days with IQR) ${ }^{d}$ & $22(12-44)$ & $14(10-22.8)$ & 0.02 \\
\hline \multicolumn{4}{|l|}{ IQR interquartile range } \\
\hline \multicolumn{4}{|c|}{ a Variables are displayed as counts and percentages in parentheses unless otherwise specified } \\
\hline \multicolumn{4}{|c|}{$\begin{array}{l}\text { b Other antimicrobials used during empiric and definitive therapy included rifampin, aminoglycosides, fluoroquinolones, macrolides, trimethoprim-sulfamethoxazole, } \\
\text { and clindamycin }\end{array}$} \\
\hline \multicolumn{4}{|c|}{$\begin{array}{l}\text { c } 6 \text { and } 9 \text { patients from the } \beta \text {-lactam plus vancomycin and } \beta \text {-lactam monotherapy subgroup respectively were excluded from the analysis due to lack of follow-up } \\
\text { blood cultures }\end{array}$} \\
\hline
\end{tabular}

Table 3 Outcome analysis comparing empiric $\beta$-lactam versus vancomycin, adjusted for age, age-adjusted Charlsoncomorbidity index, infectious diseases consultation, infective endocarditis and time to receipt of empiric antibiotics

\begin{tabular}{lllll}
\hline Outcomes & Crude OR $(\mathbf{9 5} \% \mathrm{Cl})$ & $\boldsymbol{p}$ value & Adjusted OR (95 \% Cl) & $\boldsymbol{p}$ value \\
\hline 28-day mortality & $0.60(0.23-1.55)$ & 0.29 & $0.85(0.27-2.67)$ & 0.78 \\
90-day mortality & $0.56(0.28-1.15)$ & 0.11 & $0.88(0.36-2.17)$ & 0.79 \\
\hline & Ratio of mean $(\mathbf{9 5} \% \mathrm{Cl})$ & $\boldsymbol{p}$ value & Adjusted ratio of mean $(\mathbf{9 5} \% \mathrm{Cl})$ & $\boldsymbol{p}$ value \\
\hline Duration of bacteremia & $0.77(0.64-0.93)$ & 0.01 & $0.77(0.62-0.95)$ & 0.01 \\
Hospital length-of-stay & $0.85(0.68-1.07)$ & 0.16 & $0.86(0.66-1.10)$ & 0.23 \\
\hline
\end{tabular}

Duration of bacteremia was further adjusted for surgical source control

$O R$ odds ratio, $\mathrm{Cl}$ confidence interval

among injection drug users with predominantly rightsided MSSA infective endocarditis. Even when patients were switched from vancomycin to a semi-synthetic penicillin within a median of 3 days, infection-related mortality remained high at $40.9 \%$. The overall mortality in Lodise's cohort was unusually high at $22.2 \%$ compared to a rate of $0-4 \%$ described in a systematic review by Yung et al. [25]. The largest study to date by McDanel et al. revealed that empiric $\beta$-lactam therapy (predominantly piperacillin-tazobactam and ceftriaxone) compared to vancomycin was not associated with differences in mortality in patients with MSSA bacteremia [15]. However, this study excluded patients who received empiric vancomycin plus $\beta$-lactams, did not address microbiological cure, and evaluated empiric regimens independent of the definitive antimicrobial therapy prescribed.

We included a large proportion of patients who received empiric treatment with optimal anti-MSSA agents (cloxacillin or cefazolin), whereas previous observational studies have either failed to specify the $\beta$-lactam agents used or enrolled patients who received mostly broad-spectrum $\beta$-lactams. This is an important point because not all $\beta$-lactams have equal efficacy against MSSA. As demonstrated in one study, empiric cefazolin or cloxacillin was associated with improved short-term survival compared to empiric regimens containing other $\beta$-lactams [26]. Therefore, the ideal study is one that compares a semi-synthetic anti-Staphylococcal penicillin or cefazolin to vancomycin.

Our study has several limitations. The reason for the lack of difference in the primary outcome is likely multifactorial. Because of the low event rate in both groups, our study was potentially underpowered to detect a significant difference in mortality. The lower than expected death rate may be partly due to the exclusion of patients who died within $24 \mathrm{~h}$ of the diagnosis of SAB and of patients who remained on broad-spectrum $\beta$-lactams. This group may have represented a sicker population and thus, we may have selected for less critically ill patients. The absence of matching with respect to baseline characteristics and the retrospective nature of the study may have also contributed to a lack of difference in the primary outcome. Obtaining subsequent blood cultures was often delayed, which may have led to an overestimation 
of the duration of bacteremia. However, this effect was likely balanced between both groups. Data regarding adverse effects were not collected due to the difficulty of establishing drug-related events in a retrospective study. A randomized controlled trial would be ideal to address our study question because it would provide better matching of patient baseline characteristics and control of antimicrobials prescribed, and permit prospective monitoring of adverse drug effects. In such a study, daily blood cultures would need to be collected to determine the exact date of clearance of bacteremia. As well, more accurate estimation of the expected mortality rates between groups would be needed when calculating the required sample size. Collaboration between the medical microbiology laboratory, infectious diseases service and antimicrobial stewardship team is essential to execute such a trial.

Until we have more concrete evidence from future prospective studies, the benefit of adding a $\beta$-lactam to empiric therapy for MSSA bacteremia remains unclear. Ultimately, the choice of empiric regimen will depend on patient factors, the prevalence of MRSA in the population, and the ability of the microbiology laboratory to rapidly differentiate MSSA from MRSA.

In conclusion, empiric therapy with $\beta$-lactams was associated with earlier clearance of bacteremia by a median of 1 day compared to vancomycin, but was not associated with differences in all-cause mortality or hospital LOS in patients with MSSA bacteremia. Our data should be interpreted with caution however, as major differences in the baseline characteristics between the groups may have overshadowed any potential treatment effect. Future prospective studies are needed to confirm our findings. For now, empiric treatment with vancomycin is reasonable if the prevalence of MRSA is significant. The addition of a $\beta$-lactam agent could be considered in critically ill patients.

\section{Authors's contributions \\ DW conceived and designed the study, collected and analyzed the data, wrote and revised the manuscript. TW designed the study and critically reviewed the manuscript. MR critically reviewed the manuscript. VL designed the study and critically reviewed the manuscript. All authors read and approved the final manuscript.}

\section{Author details}

${ }^{1}$ PGY-V Infectious Diseases Residency Training Program, Vancouver General Hospital, University of British Columbia, D 452 Heather Pavilion, 2733 Heather Street, Vancouver, BC V5Z 1 M9, Canada. ${ }^{2}$ Department of Pathology and Laboratory Medicine, University of British Columbia, Vancouver, BC, Canada. ${ }^{3}$ Medical Microbiology Laboratory, Division of Medical Microbiology and Infection Control, Vancouver General Hospital, JPPN1, 899 W 12th Ave., Vancouver, BC V5Z 1M9, Canada. ${ }^{4}$ Medical Microbiology Laboratory, Division of Medical Microbiology, St. Paul's Hospital, 1081 Burrard St., Vancouver, BC V6Z 1Y6, Canada.

\section{Competing interests}

The authors declare that they have no competing interests.
Funding

No external funding was required.

Received: 22 February 2016 Accepted: 17 April 2016

Published online: 26 April 2016

References

1. Van Hal SJ, Jensen SO, Vaska VL, Espedido BJ, Paterson DL, Gosbell IB. Predictors of mortality in Staphylococcus aureus bacteremia. Clin Microbiol Rev. 2012;25:362-86.

2. Thwaites GE, Edgeworth JD, Gkrania-Klotsas E, Kirby A, Tilley R, Torok ME, et al. Clinical management of Staphylococcus aureus bacteraemia. Lancet Infect Dis. 2011;11:208-22.

3. Khatib R, Saeed S, Sharma M, Riederer K, Fakih MG, Johnson LB. Impact of initial antibiotic choice and delayed appropriate treatment on the outcome of Staphylococcus aureus bacteremia. Eur J Clin Microbiol Infect Dis. 2006;25:181-5.

4. Paul M, Kariv G, Goldberg E, Raskin M, Shaked H, Hazzan R, et al. Importance of appropriate empirical antibiotic therapy for methicillin-resistant Staphylococcus aureus bacteraemia. J Antimicrob Chemother. 2010;65:2658-65.

5. David MZ, Daum RS. Community-associated methicillin-resistant Staphylococcus aureus: epidemiology and clinical consequences of an emerging epidemic. Clin Microbiol Rev. 2010;23:616-87.

6. Lodise TP, McKinnon PS, Swiderski L, Rybak M. Outcomes analysis of delayed antibiotic treatment for hospital-acquired Staphylococcus aureus bacteremia. Clin Infect Dis. 2003:36:1418-23.

7. Marchaim D, Kaye KS, Fowler VG, Anderson DJ, Chawla V, Golan Y. Case-control study to identify factors associated with mortality among patients with methicillin-resistant Staphylococcus aureus bacteraemia. Clin Microbiol Infect. 2010;16:747-52.

8. Schweizer ML, Furuno JP, Harris AD, Johnson JK, Shardell MD, McGregor JC. Empiric antibiotic therapy for Staphylococcus aureus bacteremia may not reduce in-hospital mortality: a retrospective cohort study. PLoS One. 2010;5:e11432.

9. Kim SH, Kim KH, Kim HB, Kim NJ, Kim EC, Oh MD, et al. Outcome of vancomycin treatment in patients with methicillin-susceptible Staphylococcus aureus bacteremia. Antimicrob Agents Chemother. 2008;52:192-7.

10. Lee S, Choe PG, Song KH, Park SW, Kim HB, Kim NJ, et al. Is cefazolin inferior to nafcillin for treatment of methicillin-susceptible Staphylococcus aureus bacteremia? Antimicrob Agents Chemother. 2011;55:5122-6.

11. Liu C, Bayer A, Cosgrove SE, Daum RS, Fridkin SK, Gorwitz RJ, et al. Clinical practice guidelines by the Infectious Diseases Society of America for the treatment of methicillin-resistant Staphylococcus aureus infections in adults and children. Clin Infect Dis. 2011;52:1-38.

12. Chang FY, Peacock JE, Musher DM Jr, Triplett P, MacDonald BB, Mylotte JM, et al. Staphylococcus aureus bacteremia recurrence and the impact of antibiotic treatment in a prospective multicenter study. Medicine. 2003:82:333-9.

13. Chan KE, Warren HS, Thadhani RI, Steele DJR, Hymes JL, Maddux FW, et al. Prevalence and outcomes of antimicrobial treatment for Staphylococcus aureus bacteremia in outpatients with ESRD. J Am Soc Nephrol. 2012;23:1551-9.

14. Stryjewski ME, Szczech LA, Benjamin DK Jr, Inrig JK, Kanafani ZA, Engemann JJ, et al. Use of vancomycin or first-generation cephalosporins for the treatment of hemodialysis-dependent patients with MSSA bacteremia. Clin Infect Dis. 2007;44:190-6.

15. McDanel JS, Perencevich EN, Diekema DJ, Herwaldt LA, Smith TC, Chrischilles EA, et al. Comparative effectiveness of beta-lactams versus vancomycin for treatment of methicillin-susceptible Staphylococcus aureus bloodstream infections among 122 hospitals. Clin Infect Dis. 2015;61:361-7.

16. Schweizer ML, Furuno JP, Harris AD, Johnson JK, Shardell MD, McGregor $J C$, et al. Comparative effectiveness of nafcillin or cefazolin versus vancomycin in methicillin-susceptible Staphylococcus aureus bacteremia. BMC Infect Dis. 2011;11:279.

17. Lodise TP Jr, McKinnon PS, Levine DP, Rybak MJ. Impact of empiricaltherapy selection on outcomes of intravenous drug users with 
infective endocarditis caused by MSSA. Antimicrob Agents Chemother. 2007;51:3731-3.

18. McConeghy KW, Bleasdale SC, Rodvold KA. The empirical combination of vancomycin and a $\beta$-lactam for staphylococcal bacteremia. Clin Infect Dis. 2013;57:1760-5

19. Li JS, Sexton DJ, Mick N, Nettles R, Fowler VG Jr, Ryan T, et al. Proposed modifications to the duke criteria for the diagnosis of infective endocarditis. Clin Infect Dis. 2000;30:633-8.

20. Firth D. Bias reduction of maximum likelihood estimates. Biometrika. 1993;80:27-8.

21. Schneeweiss S, Wang PS, Avorn J, Glynn RJ. Improved comorbidity adjustment for predicting mortality in Medicare populations. Health Serv Res. 2003;38:1103-20

22. Cosgrove SE, Sakoulas G, Perencevich EN, Schwaber MJ, Karchmer AW, Carmeli Y. Comparison of mortality associated with methicillin-resistant and methicillin-susceptible Staphylococcus aureus bacteremia: a metaanalysis. Clin Infect Dis. 2003;36:53-9.
23. Bai AD, Showler A, Burry L, Steinberg M, Ricciuto DR, Fernandes T, et al. Comparative effectiveness of cefazolin versus cloxacillin as definitive antibiotic therapy for MSSA bacteraemia: results from a large multicentre cohort study. J Antimicrob Chemother. 2015;70:1539-46.

24. Yilmaz M, Elaldi N, Balkan II, Arslan F, Batırel AA, Bakıcı MZ, et al. Mortality predictors of Staphylococcus aureus bacteremia: a prospective multicenter study. Ann Clin Microbiol Antimicrob. 2016;15:7. doi:10.1186/ S12941-016-0122-8.

25. Yung D, Kottachchi D, Neupane B, Haider S, Loeb M. Antimicrobials for right-sided endocarditis in intravenous drug users: a systematic review. J Antimicrob Chemother. 2007;60:921-8.

26. Paul M, Zemer-Wassercug N, Talker O, Lishtzinsky Y, Lev B, Samra Z, et al. Are all beta-lactams similarly effective in the treatment of methicillinsensitive Staphylococcus aureus bacteraemia? Clin Microbiol Infect. 2011;17:1581-6.

\section{Submit your next manuscript to BioMed Central and we will help you at every step:}

- We accept pre-submission inquiries

- Our selector tool helps you to find the most relevant journal

- We provide round the clock customer support

- Convenient online submission

- Thorough peer review

- Inclusion in PubMed and all major indexing services

- Maximum visibility for your research

Submit your manuscript at www.biomedcentral.com/submit 